\title{
Evaluación del desempeño zootécnico de alevinos de Piaractus brachypomus alimentados con dietas que aporten diferentes niveles de energía
}

\author{
Evaluation of the zootechnic performance of Piaractus brachypomus \\ fry feded with diets that provides different levels of energy.
}

\begin{abstract}
Avaliação do desempenho zootécnico de alevinos de Piaractus brachypomus alimentados com dietas que aportem diferentes níveis de energia.
\end{abstract}

Sandra L Martínez-Castillo ${ }^{1}$, Z; Luis G Quintero -Pinto ${ }^{2}$; Leidy J Peña -Osorio ${ }^{3}$

1 Z; Grupo de investigación Producción y Nutrición Animal. Corporación Educativa Nacional, Bogotá, Colombia.
2 Zoot, MSc, PhD; Profesor asociado, Universidad Nacional de Colombia. Bogotá, Colombia. Grupo de investigación
Biodiversidad y Acuicultura, Profesor asociado, Universidad Nacional de Colombia. Bogotá, Colombia.
3 Tec. Vet, Estudiante Medicina Veterinaria, U.D.C.A, Grupo de investigación Producción y Nutrición Animal. Corporación
Educativa Nacional, Bogotá, Colombia.
Email: s.martinez@cen.edu.co

Recibido: 16 de marzo de 2017

Aceptado: 23 de octubre de 2018

\section{Resumen}

Este experimento tuvo como objetivo evaluar el desempeño productivo de alevinos de Piaractus brachypomus, al ser alimentados con dietas isoproteicas (34\%) con siete niveles de energía bruta (EB) (3750, 3865, 3980, 4095, 4210, 4325, 4440 (Kcal/kg) con las siguientes relaciones de energía / proteína: 11,03; 11,37; 11,74; 12,04; 12,38; 12,72 y 13,06 Kcal EB/g PB. El diseño experimental fue completamente aleatorizado, con cuatro repeticiones. El experimento tuvo una duración de 60 días. Se usó un lote de 280 alevinos (peso medio inicial de 1,89 \pm 0,14 g), en número de 10 peces por acuario. Se evalúo el efecto de las dietas en cuanto a peso final (PF), ganancia de peso (GP), consumo de alimento (CA), tasa de conversión alimenticia (TCA), tasa de crecimiento específica (TCE), consumo de proteína (CP), tasa de eficiencia proteica (TEP), consumo de energía (CE), tasa de eficiencia energética (TEE) y un análisis de carcasas del 10\% de la población al día 60 del experimento. Finalmente, los datos fueron sometidos a un análisis de varianza (ANOVA). Se concluyó que el nivel mínimo de energía que atiende los requerimientos de esta especie es de $3980 \mathrm{Kcal} / \mathrm{Kg}$, con una relación de EB: PB de 11,74 Kcal de $\mathrm{EB} / \mathrm{g}$ de $\mathrm{PB}$, siendo semejante estadísticamente $(\mathrm{P}>0,05)$ con los niveles superiores probados.

Palabras clave: Nutrición de alevinos, Piaractus brachypomus, energía, ganancia de peso.

\begin{abstract}
The purpose of this experiment was to evaluate the productive performance of Piaractus brachypomus fry, fed with isoproteic diets (34\%) with seven levels of gross energy (EB) $(3750,3865,3980,4095,4210,4325,4440 \mathrm{Kcal} / \mathrm{kg})$ with the
\end{abstract}


next relations of gross energy / crude protein: 11,03; 11,37; 11,74; 12,04; 12,38; 12,72 and 13,06 Kcal EB / g PB. The experimental design was completely random, with four repetitions. The experiment lasted 60 days. A batch of 280 fry were used (initial average weight 1,89 $\pm 0,14 \mathrm{~g}$ ) in number of 10 fishes per aquarium. The effect of the diets was evaluated in terms of final weight (PF), weight gain (GP), feed intake (CA), feed conversion rate (TCA), specific growth rate (TCE), protein consumption (CP), protein efficiency ratio (TEP), energy consumption (CE), energy efficiency rate (TEE) and an analysis carcasses of $10 \%$ of the population on day 60 of the experiment. Finally, the data were subjected in a variance analysis (ANOVA). It concluded that the minimum level of energy that meets the requirements of this specie is $3980 \mathrm{Kcal} / \mathrm{kg}$ with a ratio of EB: PB 11,74 EB kcal / g of PB being statistically $(\mathrm{P}>0,05)$ similar to higher levels tested.

Keywords: Fry nutrition, Piaractus brachypomus, energy, weight gain.

\section{Resumo}

Esse experimento teve por objetivo avaliar o desempenho produtivo de alevinos de Piaractus brachypomus, sendo arraçoados com dietas isoproteicas (34\%) com sete níveis de energia bruta (EB) (3750, 3865, 3980, 4095, 4210,4325 e 4440 $\mathrm{Kcal} / \mathrm{kg}$ ) com as seguintes relações de energia bruta / proteína bruta: 11,$03 ; 11,37 ; 11,74 ; 12,04 ; 12,38 ; 12,72$ e 13,06 Kcal EB/g PB. O delineamento experimental foi inteiramente casualiado, com quatro repetições. O experimento teve duração de 60 dias. Empregou-se um lote de 280 alevinos (peso médio de 1,89 £0,14 g) em número de dez peixes por aquário. Avaliou-se o efeito das dietas quanto ao peso final (PF), o ganho de peso (GP), o consumo de alimento (CA), a conversão alimentar (TCA), a taxa de crescimento específica (TCE), consumo de proteína (CP), taxa de eficiência proteica (TEP), consumo de energia (CE), taxa de eficiência energética (TEE) e uma análise das carcaças de $10 \%$ da população no dia 60 do experimento. Finalmente os dados foram submetidos a uma análise de variância (ANOVA). Concluiu-se que o nível de mínimo energia que atende as exigências da espécie é de $3980 \mathrm{Kcal} / \mathrm{Kg}$, com uma relação de EB:PB de 11,74 Kcal de EB/g de $\mathrm{PB}$, sendo estatisticamente semelhantes $(\mathrm{P}>0,05)$ com os níveis superiores testados.

Palavras chave: Nutrição de alevinos, Piaractus brachypomus, energia, ganho de peso.

\section{Introducción}

La acuicultura en Colombia tiene un buen ritmo de crecimiento, con una rentabilidad mayor que las actividades agropecuarias tradicionales (FAO, 2005). De hecho, el último reporte de la producción pesquera histórica en Colombia por parte de la AUNAP registró una producción total de 150,465 toneladas de pescado atribuidas en un $70,9 \%$ a la acuicultura en el año 2015 (AUNAP, 2018).

A nivel productivo, la estabilidad de los precios de venta del producto, el aumento del costo de los insumos, especialmente del alimento (FAO, 2005), la continua expansión y mejoramiento de la industria acuícola, exige permanentes avances relacionados con la formulación y fabricación de dietas balanceadas de bajo costo y alta eficiencia para promover máximo crecimiento en el menor tiempo posible (Vásquez et al., 2002) debido a que en sistemas de cultivo intensivo los gastos por cuenta de alimento pueden oscilar entre el 75 al $85 \%$ del total de los costos de producción (Gutiérrez y Vásquez, 2008).

En la acuicultura continental de Colombia las especies con mayor volumen de oferta son: tilapia (Oreochromis spp.), trucha (Salmo trutta) y cachama blanca (Piaractus brachypomus) (AUNAP, 2018), siendo la tilapia la especie más utilizada seguida de la cachama blanca, la cual es una especie oriunda de las cuencas de los ríos Orinoco y Amazonas en América del sur (Gon- zalez, 2001), perteneciente al orden Characiforme y a la familia Serrasalmidae (Froese y Pauly, 2019), con hábitos alimenticios omnívoros tendiendo a frugívoro, destacándose por presentar importantes condiciones de cultivo tales como rápido crecimiento, uniformidad de talla, resistencia al manejo y a las enfermedades (González, 2001).

En el cultivo comercial de $P$. brachypomus en estanques y jaulas se necesita una disponibilidad regular de alevinos para crianza y una dieta que cubra sus requerimientos nutricionales, especialmente en proteína y energía (Gutiérrez et al., 2010). Sin embargo, no existen raciones formuladas específicamente esta especie, razón por la cual se recurre al uso de concentrados diseñados para otras especies como tilapia o trucha. No obstante, estos concentrados no permiten a la cachama expresar todo su potencial de crecimiento, ya que no están formulados con base en las cualidades digestivas (Vásquez, Yossa, y Gutiérrez, 2013).

Dentro de la formulación, la proteína es uno de los nutrientes más importantes que afecta el rendimiento piscícola (Gutiérrez et al., 2010), pero a su vez es uno de los componentes más costosos en la dieta (Cho et al., 2005; Craig y McLean, 2005; Miller et al., 2005). Es importante que la proteína sea utilizada para la síntesis del músculo y tejidos, y no para el metabolismo energético, si se quiere rentabilizar la dieta (Williams et al., 2003; Ozorio et al., 2006). 
El nivel de energía también es crítico, debido a que altos niveles de energía en la dieta pueden reducir el consumo de alimento y la ingesta de nutrientes necesarios para obtener un buen crecimiento y, por ende, un excelente rendimiento. Por otro lado, bajos niveles de energía en la dieta pueden causar que la proteína deba ser usada como fuente de energía para satisfacer los requerimientos energéticos para el metabolismo basal de los peces, en lugar de ser usada para el crecimiento. Por lo tanto, la proteína dietaría y los niveles de energía deben estar en un balance adecuado para optimizar la producción piscícola (Gutiérrez et al., 2010).

Una inadecuada relación entre proteína y energía en la dieta conduce a un aumento de los costos de producción de peces y el deterioro de la calidad del agua resultante del desperdicio del alimento (Lee y Kim, 2005).

Por lo tanto, el objetivo del presente estudio fue evaluar el desempeño de los alevinos de $P$. brachypomus, suministrando la alimentación con siete dietas isoproteicas $(34 \%)$, con diferentes niveles de energía (3750, 3865, 3980, 4095, 4210, 4325, $4440 \mathrm{Kcal} / \mathrm{kg}$ ), para determinar la mejor relación de energía bruta / proteína bruta, que permita suplir los requerimientos nutricionales de los alevinos, optimizando el rendimiento en cultivo y mejorando la ganancia de peso y conversión alimenticia.

\section{Materiales y métodos}

Este experimento fue realizado en el Laboratorio de Ictiología de la Facultad de Medicina Veterinaria y Zootecnia de la Universidad Nacional de Colombia, sede Bogotá. Se utilizaron 28 acuarios de fibra de vidrio con capacidad para 80 litros de agua dotados de aireación, flujo continuo de agua, filtro biológico para remover del agua la excretas de los peces y eventuales excesos de ración del fondo de esos acuarios y control de temperatura del agua.

Quincenalmente se realizó un control de calidad de agua $(\mathrm{pH}$, oxígeno disuelto, amonio, dureza, alcalinidad y nitritos) con el kit multiparámetros de agua Hach $^{\circledR}$ modelo FF1A, en tres estanques al azar y se efectuaba un recambio del $80 \%$ en la totalidad de acuarios para permitir la reducción de las concentraciones de amonio y nitritos.

El diseño fue completamente al azar, con siete niveles de energía bruta $(3750,3865,3980,4095,4210$, 4325,4440 Kcal EB/ kg de ración), un nivel de proteína (34\%) y cuatro repeticiones.
Las dietas fueron elaboradas según las normas presentadas por National Research Council (1993). La formulación se realizó de tal forma que presentaron relaciones de energía bruta / proteína bruta de: 11,03; 11,$37 ; 11,74 ; 12,04 ; 12,38 ; 12,72$ y $13,06 \mathrm{Kcal} \mathrm{EB} / \mathrm{g}$ PB.

La elaboración de las dietas se realizó en el Laboratorio de Nutrición de la Universidad Nacional, sede Bogotá y para ello se utilizaron ingredientes de uso común en la industria de raciones balanceadas para peces en general cuya composición es presentada en la Tabla 1 y contenido nutricional en la Tabla 2. Los ingredientes secos fueron molidos para obtener un grano más fino y homogeneizado en las proporciones respectivas, posteriormente cuando ya toda la mezcla pasó por el molino se llevó a un platón, se agregaron las cantidades formuladas de aceite, fue extruido, procesado para obtener pellets con el diámetro apropiado para el tamaño de los alevinos y sometido a un proceso de secado en el horno para finalmente ser almacenado en bolsas herméticas de plástico.

El experimento tuvo una duración de 60 días. 7 días antes del inicio de la fase experimental, los peces fueron sometidos a un periodo de adaptación ambiental y manejo. Se empleó un lote de 280 alevinos de Piaractus brachypomus. Estos fueron distribuidos aleatoriamente, con peso inicial medio de 1,89 $\pm 0,14$ g en número de 10 individuos por acuario. Los peces de los diferentes tratamientos se alimentaron ad libitum, tres veces al día 8:00, 12:00 y 16:00 horas en niveles de posible saciedad bajo un fotoperiodo de 12 horas luz.

Todos los individuos de cada tratamiento fueron pesados al inicio y posteriormente cada 15 días en balanza analítica con aproximación de 0,01g completando un total de 4 pesajes. Siguiendo las metodologías de Hafedh (1999), Bacconi (2002) y Vásquez (2004) se evaluaron las siguientes variables: Peso final (PF); Ganancia de peso (GP); Consumo de alimento (CA); Tasa de crecimiento específica (TCE), tasa de conversión alimenticia (TCA); Consumo de proteína (CP);Tasa de eficiencia proteica (TEP); Consumo de energía (CE) , Tasa de eficiencia energética (TEE) y un análisis de carcasas del $10 \%$ de la población.

- GP: Peso final - peso inicial

- CA: Peso inicial del alimento - peso final del alimento

- TCE: (log peso final - log peso inicial / tiempo de experimento días) x100

- TCA: Consumo de alimento/ Ganancia de peso 
Tabla 1. Formulación de las dietas experimentales para alevinos Piaractus brachypomus con distintos niveles de energía.

\begin{tabular}{|c|c|c|c|c|c|c|c|}
\hline \multirow{2}{*}{ Ingrediente } & \multicolumn{7}{|c|}{ Tratamiento } \\
\hline & 1 & 2 & 3 & 4 & 5 & 6 & 7 \\
\hline Pescado, harina $(67 \%)$ & 120,00 & 129,00 & 138,00 & 147,01 & 156,01 & 165,01 & 174,01 \\
\hline Soya, torta $(46 \%)$ & 484,14 & 419,50 & 354,85 & 290,21 & 225,57 & 160,92 & 96,28 \\
\hline Haina de carne $(61 \%)$ & 0,00 & 13,33 & 26,67 & 40,00 & 53,33 & 66,67 & 80,00 \\
\hline Maíz, Gluten (60\%) & 0,00 & 23,33 & 46,67 & 70,00 & 93,33 & 116,67 & 140,00 \\
\hline Trigo Tercera & 100,00 & 91,67 & 83,33 & 75,00 & 66,67 & 58,33 & 50,00 \\
\hline Maíz, harina & 0,00 & 54,80 & 109,61 & 164,41 & 219,21 & 274,01 & 328,82 \\
\hline Arroz, quebrado & 225,09 & 195,91 & 166,73 & 137,54 & 108,36 & 79,18 & 50,00 \\
\hline Aceite pescado & 5,46 & 4,55 & 3,64 & 2,73 & 1,82 & 0,91 & 0,00 \\
\hline Aceite soya & 0,00 & 7,43 & 14,87 & 22,30 & 29,74 & 37,17 & 44,60 \\
\hline L- Lisina HCL & 0,00 & 0,67 & 1,33 & 2,00 & 2,67 & 3,33 & 4,00 \\
\hline DL-Met & 1,09 & 0,91 & 0,73 & 0,55 & 0,36 & 0,18 & 0,00 \\
\hline L-Treo & 0,00 & 0,00 & 0,00 & 0,00 & 0,00 & 0,00 & 0,00 \\
\hline L-Trip & 0,00 & 0,00 & 0,00 & 0,00 & 0,00 & 0,00 & 0,00 \\
\hline Premix & 5,00 & 5,00 & 5,00 & 5,00 & 5,00 & 5,00 & 5,00 \\
\hline Sal & 2,00 & 2,00 & 2,00 & 2,00 & 2,00 & 2,00 & 2,00 \\
\hline Fos bical & 34,09 & 30,51 & 26,92 & 23,34 & 19,75 & 16,17 & 12,59 \\
\hline $\mathrm{CaCO} 3$ & 10,42 & 8,68 & 6,95 & 5,21 & 3,47 & 1,74 & 0,00 \\
\hline BHT & 0,20 & 0,20 & 0,20 & 0,20 & 0,20 & 0,20 & 0,20 \\
\hline Vit C (35\%) & 1,43 & 1,43 & 1,43 & 1,43 & 1,43 & 1,43 & 1,43 \\
\hline Clor. Colina (70\%) & 1,07 & 1,07 & 1,07 & 1,07 & 1,07 & 1,07 & 1,07 \\
\hline CMC & 10,00 & 10,00 & 10,00 & 10,00 & 10,00 & 10,00 & 10,00 \\
\hline Total & 1000,00 & 1000,0 & 1000,0 & 1000,0 & 1000,0 & 1000,0 & 1000,0 \\
\hline
\end{tabular}

- CP: Alimento consumido (g) * porcentaje de proteína en la dieta $/ 100$

- TEP: Ganancia de peso / Proteína bruta consumida.

- CE: Alimento consumido $(\mathrm{g})^{*}$ niveles de energía de la dieta (Kcal)

- TEE: Consumo de alimento/ consumo de energía

\section{Análisis estadísticos}

Debido a que el trabajo fue de tipo experimental, los resultados se analizaron a través de la metodología Análisis de Varianza (ANOVA) y la comparación de medias con la prueba Duncan, utilizando el programa SAS ${ }^{\circledR}$. Con un nivel de significancia de $P<0,05$. Se verificaron los supuestos de normalidad y homogeneidad de varianzas.

\section{Aspectos bioéticos}

Los animales fueron adquiridos de la estación piscícola "Agua Linda" ubicada en Cumaral Meta. De allí fueron transportados en bolsas plásticas provistas de agua de buena calidad y con la incorporación de oxígeno. Se transportaron máximo 200 alevinos por bolsa, siguiendo el protocolo de empaque y transporte de la empresa.

Al día 60 los animales utilizados para el análisis de carcasas fueron insensibilizados mediante choque térmico bajando temperatura y posterior sección de la médula espinal, siguiendo las normas de bioética para la experimentación con animales, considerando la Ley 84 del 27 de diciembre de 1989 "Por la cual se adopta el estatuto Nacional de Protección de los Animales". 
Tabla 2. Valor nutricional de las dietas experimentales para Piaractus brachypomus en estudio.

\begin{tabular}{|c|c|c|c|c|c|c|c|c|}
\hline \multirow{2}{*}{\multicolumn{2}{|c|}{ Nutriente }} & \multicolumn{7}{|c|}{ Tratamiento } \\
\hline & & \multirow{2}{*}{$\begin{array}{c}1 \\
92,9 \\
\end{array}$} & \multirow{2}{*}{$\begin{array}{c}2 \\
92,7 \\
\end{array}$} & \multirow{2}{*}{$\begin{array}{c}3 \\
92,5 \\
\end{array}$} & \multirow{2}{*}{$\begin{array}{c}\mathbf{4} \\
92,4 \\
\end{array}$} & \multirow{2}{*}{$\begin{array}{c}\mathbf{5} \\
92,2 \\
\end{array}$} & \multirow{2}{*}{$\begin{array}{c}6 \\
92,0 \\
\end{array}$} & \multirow{2}{*}{$\begin{array}{c}7 \\
91,9\end{array}$} \\
\hline Materia Seca & $\%$ & & & & & & & \\
\hline Cantidad usada & $\mathrm{Kg}$ & 1,00 & 1,00 & 1,00 & 1,00 & 1,00 & 1,00 & 1,00 \\
\hline Proteína Bruta & $\%$ & 34,31 & 34,26 & 34,21 & 34,15 & 34,10 & 34,05 & 34,00 \\
\hline Energía Bruta & $\mathrm{Kcal} / \mathrm{kg}$ & 3750 & 3865 & 3980 & 4095 & 4210 & 4325 & 4440 \\
\hline Energía Digestible & $\mathrm{Kcal} / \mathrm{kg}$ & 3199 & 3280 & 3361 & 3443 & 3524 & 3606 & 3687 \\
\hline Fibra Bruta & $\%$ & 2,49 & 2,39 & 2,29 & 2,19 & 2,09 & 1,99 & 1,89 \\
\hline Extracto Etéreo & $\%$ & 3,08 & 4,07 & 5,05 & 6,04 & 7,03 & 8,01 & 9,00 \\
\hline Almidón & $\%$ & 20,00 & 21,42 & 22,83 & 24,25 & 25,67 & 27,08 & 28,50 \\
\hline Calcio & $\%$ & 1,80 & 1,76 & 1,71 & 1,67 & 1,63 & 1,59 & 1,54 \\
\hline P Tot & $\%$ & 1,28 & 1,23 & 1,19 & 1,14 & 1,09 & 1,05 & 1,00 \\
\hline Lisina & $\%$ & 2,04 & 2,02 & 2,00 & 1,98 & 1,96 & 1,94 & 1,92 \\
\hline Arginina & $\%$ & 2,42 & 2,32 & 2,22 & 2,11 & 2,01 & 1,91 & 1,81 \\
\hline Histidina & $\%$ & 0,86 & 0,84 & 0,82 & 0,80 & 0,78 & 0,76 & 0,74 \\
\hline Isoleucina & $\%$ & 1,53 & 1,49 & 1,45 & 1,42 & 1,38 & 1,35 & 1,31 \\
\hline Leucina & $\%$ & 2,60 & 2,73 & 2,87 & 3,01 & 3,15 & 3,29 & 3,42 \\
\hline Metionina & $\%$ & 0,70 & 0,70 & 0,69 & 0,69 & 0,68 & 0,68 & 0,67 \\
\hline Ácido aspártico & $\%$ & 1,20 & 1,18 & 1,16 & 1,14 & 1,12 & 1,11 & 1,09 \\
\hline Fenilalanina & $\%$ & 1,65 & 1,64 & 1,62 & 1,61 & 1,60 & 1,59 & 1,58 \\
\hline The & $\%$ & 1,35 & 1,33 & 1,31 & 1,29 & 1,27 & 1,25 & 1,23 \\
\hline Triptófano & $\%$ & 0,44 & 0,42 & 0,39 & 0,36 & 0,34 & 0,31 & 0,29 \\
\hline Valina & $\%$ & 1,68 & 1,66 & 1,64 & 1,62 & 1,60 & 1,58 & 1,55 \\
\hline Vitamina C & $\%$ & 0,05 & 0,05 & 0,05 & 0,05 & 0,05 & 0,05 & 0,05 \\
\hline Colina & $\%$ & 0,08 & 0,08 & 0,08 & 0,08 & 0,08 & 0,08 & 0,08 \\
\hline$n-3$ & $\%$ & 0,50 & 0,55 & 0,61 & 0,66 & 0,72 & 0,77 & 0,83 \\
\hline$n-6$ & $\%$ & 0,91 & 1,27 & 1,62 & 1,98 & 2,33 & 2,69 & 3,05 \\
\hline
\end{tabular}

\section{Resultados y discusión}

\section{Variables ambientales}

La temperatura media del agua durante todo el periodo experimental fue de $28 \pm 2^{\circ} \mathrm{C}$, el pH de 5,4 $\pm 0,17$, la concentración de oxígeno disuelto de $6 \pm 1,8 \mathrm{mg} / \mathrm{L}$, el amonio $>3 \mathrm{ppm}$ de $\mathrm{N}-\mathrm{NH}_{3}$, la dureza $85,5 \pm 8,5 \mathrm{mg}$ de $\mathrm{CaCO}_{3} / \mathrm{L}$, la alcalinidad 34,2 $\pm 0,0 \mathrm{mg}$ de $\mathrm{CaCO}_{3}$ $/$ L y los nitritos de 1,65 $\pm 0,0$. Valores considerados en el rango de confort de la especie (Quintero et al, 2011).

\section{Crecimiento}

Los valores de peso final (PF), ganancia de peso (GP) y tasa de crecimiento específica (TCE) de los alevinos de $P$. brachypomus obtenidos en el estudio, son presentados en la Tabla 3. De estos valores se puede inferir que los peces alimentados con las dietas isoproteicas (34\%) que contenían los niveles de energía entre 3980 $\mathrm{Kcal} / \mathrm{kg}$ y $4095 \mathrm{Kcal} / \mathrm{kg}$ con relación EB (kcal)/PB (g) entre 11,74 y 12,04 cumplen con los requerimientos energéticos siendo semejantes estadísticamente $(P>0,05)$ para las variables de crecimiento. 
De acuerdo con resultados obtenidos por Salinas (2018) en un estudio sobre niveles de energía y relación proteína energía en Piaractus brachypomus encontró mejores resultados en ganancia de peso con un nivel de energía digestible (ED) 3,400 Kcal/kg y un nivel de proteína del $28 \%$ reportando una tasa de crecimiento específica de 1,12\%, resultado inferior a los encontrados por Vásquez et al., (2011) evaluando diferentes niveles de proteína en alevines de paco con valores para la TCE de 2,25\% con 31,3\% de proteína. Así mismo, dichos valores son inferiores a los resultados obtenidos en el presente estudio que obtuvo estadísticamente los mejores rendimientos en cuanto a crecimiento (PF, GP y TCE) con un nivel de energía bruta de 3980 Kcal (3361 Kcal de ED) y un nivel del 34

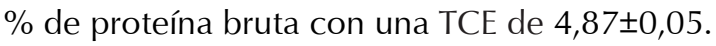

\section{Eficiencia alimenticia}

Los valores de la tasa de conversión alimenticia (TCA), tasa de eficiencia proteica (TEP) y tasa de eficiencia energía (TEE) son presentados en la Tabla 4. La mejor eficiencia de aprovechamiento de alimento se dio con un nivel de energía de $4095 \mathrm{Kcal}$ con una relación de EB/PB de 12,04, por haber presentado la menor tasa de conversión alimenticia y las mayores tasas de eficiencia proteica y energética simultáneamente.

Vásquez y Gutiérrez et al., (2010) determinó que la conversión alimenticia de alevinos de Colossoma macroporum alimentados con dietas con un rango de 25 al 33\% de proteína y un nivel de energía de 2,7 Kcal de ED fue semejante $(P>0,05)$, sin embargo al evaluar la relación de eficiencia proteica (REP) determinó que los mejores resultados se obtienen con un nivel de proteína del $25 \%$ y un nivel de energía de 2,7 Kcal de ED, adicionalmente presentó una progresiva disminución en la REP a medida que se aumentaban los niveles de proteína obteniendo valores de 3,04 $\pm 0,03$ en un nivel de 33\% proteína. Morillo et al., (2013) en alevinos de Colossoma macroporum alimentados con dietas con niveles de proteína bruta entre 28,9 y $32,5 \%$ y energía digestible (ED) teórica entre 4121 y $4300 \mathrm{kcal} / \mathrm{kg}$ reportó para la eficiencia proteica valores entre 2,49 y $2,67(P>0,05)$ los cuales son muy similares a los reportados por (Vásquez, et al., 2002) para cachama blanca alimentada con dieta con $32,3 \%$ PB, que muestran un coeficiente de eficiencia proteica (CEP) de 2,39. No obstante el presente estudio presentó valores superiores a los reportados por los autores citados, con eficiencias proteicas en un rango de 3,97 a 4,57 con un nivel de proteína de $34 \%$ y eficiencias energéticas en un rango de 0,35 a 0,41 en niveles de energía de 3750 a $4440 \mathrm{Kcal} / \mathrm{kg}$ de alimento.

Los resultados obtenidos en este estudio demostraron que a medida que se aumentaron los niveles de energía la eficiencia aumentaba exceptuando la TEE que a partir del nivel energético de $4095 \mathrm{Kcal} / \mathrm{kg}$ presentó una tendencia de disminución, sugiriendo que no es pertinente el incremento de energía después de ese nivel.

\section{Consumo}

El tratamiento con menor nivel de energía que presentó los mejores comportamientos en las variables CA y $\mathrm{CP}$ corresponde al nivel de energía de $3980 \mathrm{Kcal}$ EB/ $\mathrm{Kg}$ y una relación EB/PB de 11,74. Para el caso de la energía el mayor consumo con menor nivel de energía

Tabla 3. Peso final (PF), ganancia de peso (GP) y tasa de crecimiento específico (TCE) de alevinos de Piaractus brachypomus alimentados con dietas conteniendo diferentes niveles de energía.

\begin{tabular}{|c|c|c|c|c|c|}
\hline $\begin{array}{c}\text { Nivel de } \\
\text { proteína }\end{array}$ & $\begin{array}{c}\text { Nivel de } \\
\text { Energía }\end{array}$ & EB/PB & $\begin{array}{c}\text { PF } \\
(\mathbf{g})\end{array}$ & $\begin{array}{c}\text { GP } \\
(\mathbf{g})\end{array}$ & $\begin{array}{c}\text { TCE } \\
\mathbf{( \% )}\end{array}$ \\
\hline $34 \%$ & $3750 \mathrm{Kcal}$ & 11,03 & $32,43 \pm 3,24 \mathrm{bc}$ & $30,53 \pm 3,19 \mathrm{bc}$ & $4,72 \pm 0,16 \mathrm{~b}$ \\
\hline $34 \%$ & $3865 \mathrm{Kcal}$ & 11,37 & $32,13 \pm 3,29 \mathrm{c}$ & $29,2 \pm 3,34 \mathrm{c}$ & $4,63 \pm 0,21 \mathrm{~b}$ \\
\hline $34 \%$ & $3980 \mathrm{Kcal}$ & 11,74 & $35,94 \pm 1,54 \mathrm{abc}$ & $34,01 \pm 1,52 \mathrm{ab}$ & $4,87 \pm 0,05 \mathrm{ab}$ \\
\hline $34 \%$ & $4095 \mathrm{Kcal}$ & 12,04 & $36,31 \pm 1,76 \mathrm{abc}$ & $34,54 \pm 1,62 \mathrm{ab}$ & $5,06 \pm 0,35 \mathrm{a}$ \\
\hline $34 \%$ & $4210 \mathrm{Kcal}$ & 12,38 & $39,06 \pm 3,03 \mathrm{ab}$ & $37,18 \pm 2,97 \mathrm{a}$ & $5,05 \pm 0,08 \mathrm{a}$ \\
\hline $34 \%$ & $4325 \mathrm{Kcal}$ & 12,72 & $40,7 \pm 5,03 \mathrm{a}$ & $38,82 \pm 4,96 \mathrm{a}$ & $5,11 \pm 0,14 \mathrm{a}$ \\
\hline $34 \%$ & $4440 \mathrm{Kcal}$ & 13,06 & $40,8 \pm 1,98 \mathrm{a}$ & $38,84 \pm 1,97 \mathrm{a}$ & $5,05 \pm 0,08 \mathrm{a}$ \\
\hline
\end{tabular}

1 Los valores representan la media \pm desviación estándar. Valores con letras iguales no presentan diferencia significativa $(P>0,05)$. 
Tabla 4. Tasa de conversión alimenticia (TCA), tasa de eficiencia proteica (TEP), tasa de eficiencia energética (TEE) de alevinos de Piaractus brachypomus alimentados con dietas conteniendo diferentes niveles de energía.

\begin{tabular}{|c|c|c|c|c|c|}
\hline $\begin{array}{c}\text { Nivel de } \\
\text { proteína }\end{array}$ & $\begin{array}{c}\text { Nivel de } \\
\text { energía }\end{array}$ & EB/PB & TCA & TEP & TEE \\
\hline $34 \%$ & $3750 \mathrm{Kcal}$ & 11,03 & $0,65 \pm 0,05 \mathrm{bc}$ & $4,57 \pm 0,34 \mathrm{ab}$ & $0,41 \pm 0,03 \mathrm{a}$ \\
\hline $34 \%$ & $3865 \mathrm{Kcal}$ & 11,37 & $0,75 \pm 0,10 \mathrm{a}$ & $3,97 \pm 0,53 \mathrm{c}$ & $0,36 \pm 0,05 \mathrm{bc}$ \\
\hline $34 \%$ & $3980 \mathrm{Kcal}$ & 11,74 & $0,72 \pm 0,02 \mathrm{ab}$ & $4,09 \pm 0,14 \mathrm{bc}$ & $0,36 \pm 0,01 \mathrm{bc}$ \\
\hline $34 \%$ & $4095 \mathrm{Kcal}$ & 12,04 & $0,61 \pm 0,02 \mathrm{c}$ & $4,80 \pm 0,15 \mathrm{a}$ & $0,40 \pm 0,01 \mathrm{ab}$ \\
\hline $34 \%$ & $4210 \mathrm{Kcal}$ & 12,38 & $0,66 \pm 0,03 \mathrm{bc}$ & $4,50 \pm 0,19 \mathrm{ab}$ & $0,36 \pm 0,02 \mathrm{bc}$ \\
\hline $34 \%$ & $4325 \mathrm{Kcal}$ & 12,72 & $0,67 \pm 0,07 \mathrm{bc}$ & $4,44 \pm 0,46 \mathrm{abc}$ & $0,35 \pm 0,04 \mathrm{c}$ \\
\hline $34 \%$ & $4440 \mathrm{Kcal}$ & 13,06 & $0,64 \pm 0,03 \mathrm{bc}$ & $4,60 \pm 0,20 \mathrm{ab}$ & $0,35 \pm 0,02 \mathrm{c}$ \\
\hline
\end{tabular}

1. Los valores representan la media \pm desviación estándar. Valores con letras iguales indican que no hay una diferencia significativa $(\mathrm{P}>0,05)$.

correspondió al tratamiento con 4325 kcal y relación EB/PB de 12,72 (Tabla 5).

Salinas (2018) en su estudio productivo y corporal de Piaractus brachypomus obtuvo mejores consumos con el tratamiento con $3.4 \mathrm{Mcal} \mathrm{ED} / \mathrm{kg}$ y una relación de proteína a energía de $90 \mathrm{~g} /$ Mcal ED (11,1kcal ED/g $\mathrm{PB})$ mientras que el menor consumo se reportó con la dieta de 3.2 Mcal ED/Kg y 110 g PB/Mcal ED (154.4 $\mathrm{g} / \mathrm{pez}$ ). Dichos resultados varían en la tendencia en cuanto al consumo señalada por algunos autores quienes indican que disminuye conforme aumentan los niveles de energía en la dieta, ya que los peces, al igual que otras especies terrestres, consumen alimento para satisfacer principalmente sus requerimientos energéticos (Sampaio et al., 2000; Hernández et al., 2001).

\section{Composición corporal}

En la Tabla 6 se muestran los resultados del análisis bromatológico de los alevinos de $P$. brachypomus a nivel de materia seca $(\mathrm{MS})$, proteína bruta $(\mathrm{PB})$, energía bruta $(E B)$, proteína retenida $(P R)$, porcentaje del índice de retención corporal de proteína (IRCPR), energía retenida (ER) y porcentaje del índice de retención corporal de energía (IRCER).

Según los valores de retención de proteína (PR) se puede inferir que fue semejante para todos los tratamientos debido a que en la formulación de las dietas se manejó un nivel isoproteico (34\%); sin embargo, en la variable de energía (ER) se observa que la retención es mayor en aquellos tratamientos con un nivel superior o igual a $3990 \mathrm{Kcal} / \mathrm{kg}$ de alimento.

Tabla 5. Consumo de alimento (CA), consumo de proteína (CP), consumo de energía (CE) de alevinos de Piaractus brachypomus alimentados con dietas conteniendo diferentes niveles de energía.

\begin{tabular}{|c|c|c|c|c|c|}
\hline $\begin{array}{c}\text { Nivel de } \\
\text { proteína }\end{array}$ & $\begin{array}{c}\text { Nivel de } \\
\text { energía }\end{array}$ & EB/PB & CA & CP & CE \\
\hline $34 \%$ & $3750 \mathrm{Kcal}$ & 11,03 & $53,79 \pm 1,56 \mathrm{~d}$ & $6,68 \pm 0,49 \mathrm{~d}$ & $73,64 \pm 5,41 \mathrm{e}$ \\
\hline $34 \%$ & $3865 \mathrm{Kcal}$ & 11,37 & $85,9 \pm 1,97 \mathrm{~b}$ & $7,38 \pm 0,23 \mathrm{c}$ & $81,69 \pm 2,57 \mathrm{~d}$ \\
\hline $34 \%$ & $3980 \mathrm{Kcal}$ & 11,74 & $89,68 \pm 0,95 \mathrm{a}$ & $8,31 \pm 0,16 \mathrm{ab}$ & $95,08 \pm 1,81 \mathrm{c}$ \\
\hline $34 \%$ & $4095 \mathrm{Kcal}$ & 12,04 & $64,49 \pm 1,38 \mathrm{c}$ & $7,19 \pm 0,14 \mathrm{c}$ & $86,57 \pm 1,68 \mathrm{~d}$ \\
\hline $34 \%$ & $4210 \mathrm{Kcal}$ & 12,38 & $90,03 \pm 2,18 \mathrm{a}$ & $8,26 \pm 0,33 \mathrm{~b}$ & $102,3 \pm 4,13 \mathrm{~b}$ \\
\hline $34 \%$ & $4325 \mathrm{Kcal}$ & 12,72 & $92,76 \pm 0,57 \mathrm{a}$ & $8,74 \pm 0,29 \mathrm{a}$ & $111,16 \pm 3,66 \mathrm{a}$ \\
\hline $34 \%$ & $4440 \mathrm{Kcal}$ & 13,06 & $91,74 \pm 0,22 \mathrm{a}$ & $8,45 \pm 0,17 \mathrm{ab}$ & $110,35 \pm 2,25 \mathrm{a}$ \\
\hline
\end{tabular}

1. Los valores representan la media \pm desviación estándar para CA, CP y CE. Valores con letras iguales indican que no hay una diferencia significativa $(\mathrm{P}>0,05)$ 
Tabla 6. Análisis bromatológico de alevinos de P. brachypomus en alimentados con dietas conteniendo diferentes niveles de energía.

\begin{tabular}{|c|c|c|c|c|c|c|c|}
\hline $\begin{array}{c}\text { Niveles de } \\
\text { energía }\end{array}$ & MS & PB (\%) & $\begin{array}{c}\text { EB }(\mathbf{M c a l} / \\
\mathbf{k g})\end{array}$ & PR (\%) & IRCPR (\%) & $\begin{array}{c}\text { ER (Mcal/ } \\
\text { kg) }\end{array}$ & IRCER (\%) \\
\hline Inicial & 89 & 68,7 & 4,6 & & & & \\
\hline $3750 \mathrm{Kcal}$ & 90 & 65,1 & 5,5 & 19,8 & 100 & 1,7 & 100 \\
\hline $3865 \mathrm{Kcal}$ & 91 & 65,0 & 5,7 & 18,9 & 95 & 1,7 & 100 \\
\hline $3980 \mathrm{Kcal}$ & 92 & 60,1 & 5,8 & 20,3 & 103 & 2,0 & 118 \\
\hline $4095 \mathrm{Kcal}$ & 93 & 58,2 & 6,0 & 19,9 & 101 & 2,1 & 124 \\
\hline $4210 \mathrm{Kcal}$ & 93 & 55,6 & 6,1 & 20,4 & 103 & 2,3 & 135 \\
\hline $4325 \mathrm{Kcal}$ & 93 & 56,2 & 6,1 & 21,6 & 109 & 2,4 & 141 \\
\hline $4450 \mathrm{Kcal}$ & 93 & 53,7 & 6,2 & 20,6 & 104 & 2,4 & 141 \\
\hline
\end{tabular}

Allan y Booth (2004), mencionan que los niveles de los nutrientes no deben ser tan altos para optimizar su retención; Vinicius y Machado (2002), mencionan que niveles de $3000 \mathrm{kcal}$ de energía son suficientes en las dietas de Brycon orbignyanus, mientras que Kim et al., (2004) reportan que para juveniles de Paralichthys olivaceus la mejor tasa de retención de nutrientes se obtiene al utilizar una dieta con $45 \%$ de proteína y 3990 kcal de energía digestible. Para este estudio se observa que con un nivel de $34 \%$ de proteína y una energía bruta mayor de $3990 \mathrm{Kcal}$, recomendado en las variables analizadas anteriormente, mantiene una buena retención de nutrientes.

\section{Conclusiones}

En las condiciones experimentales del presente estudio, suministrando dietas con $34 \%$ de proteína bruta y diferentes niveles de energía, se puede concluir que el nivel mínimo de energía bruta que atiende los requerimientos de los alevinos de P. brachypomus con óptimo desempeño zootécnico fue de $3980 \mathrm{Kcal} / \mathrm{kg}$, correspondiendo a la relación de 11,74 Kcal de EB/g $\mathrm{PB}$, contenidos en $1 \mathrm{~kg}$ de alimento.

\section{Agradecimientos}

Los autores agradecen al Centro de Investigaciones de la Corporación Educativa Nacional (CID-CEN), al Laboratorio de Ictiología y de Peces Ornamentales de la Universidad Nacional de Colombia, así como a los integrantes del grupo de Investigación de nutrición y producción animal de la Corporación Educativa Nacional Gaona Osorio Jonathan Alexander, González Pinzón Michel Estefanía ,Monrroy Chaparro Irene, Niampira Quintero Cristian David, Rojas Castellanos
Stephanie y Sánchez Saavedra Giseth Tatiana por su colaboración.

\section{Bibliografía}

Allan G, Booth M. Effects of extrusion on digestibility of peas, Lupins canola meal and soybean meal in silver perch Blyanus biidyanus (mitchell) diets. Aquacult. Res. 2004;35:981-991.

AUNAP. 2018. Acuicultura en colombia. Retrieved from https:// www.aunap.gov.co/images/convenio/presentacion-tecnicaacuicultura-en-colombia.pdf

Bacconi D. 2002. Requerimiento nutricional de vitamina A para los alevines de tilapia negra Oreochromis niloticus. Tesis de maestría en agronomía, área de investigación de la ciencia y el pastoreo de animales, Inglaterra: Escuela Superior de Agricultura Luiz de Queiroz de la Universidad de São Paulo.

Cho S, Lee S, Lee J. Effect of dietary protein and lipid levels on growth and body composition of juvenile turbot (Scophthalmus maximus) reared under optimum salinity and temperature condition. Aquacult Nutr. 2005;11:235-240.

Craig S, McLean E. 2005. The organic movement: a role for NuProR as an alternative protein source. In Jacques K, Lyons TP (Eds.) Nutritional Biotechnology in the Food and Feed Industry. Nottingham University Press. Nottingham, UK. 286-293 p.

FAO. 2005-2019. National Aquaculture Sector Overview. Visión general del sector acuícola nacional - Colombia. National Aquaculture Sector Overview Fact Sheets. Texto de Salazar Ariza, G. In: Departamento de Pesca y Acuicultura de la FAO [en línea]. Roma. Actualizado 1 February 2005. [Citado 10 April 2019].

Froese R, Pauly D. (eds). 2019. FishBase (version Feb 2018). In: Species 2000 \& ITIS Catalogue of Life, 25th March 2019 (Roskov Y., Ower G., Orrell T., Nicolson D., Bailly N., Kirk P.M., Bourgoin T., DeWalt R.E., Decock W., Nieukerken E. van, Zarucchi J., Penev L., eds.). Digital resource at www.catalogueoflife.org/ col. Species 2000: Naturalis, Leiden, the Netherlands. ISSN 2405-8858. 
González R. 2001. El cultivo de cachama (pp. 329-345). In: H. Rodríguez, P. V. Daza, \& M. Carrillo (Eds.), Fundamentos de acuicultura continental Bogotá, DC: Instituto Nacional de Pesca y Acuicultura. INPA, Bogotá, Colombia, 469p.

Gutiérrez FW, Quispe M, Valenzuela L, Contreras G, Zaldívar J .2010. Utilización de la proteína dietaria por alevinos de la gamitana, Colossoma macropomum, alimentados con dietas isocalóricas. Rev. Peru. Biol.17: 219-223 p.

Gutiérrez M,Vásquez W. Digestibilidad de glicine max I, soya, en juveniles de cachama blanca Piaractus brachypomus Cuvier 1818. Orinoquia. 2008;12(2):141-148.

Hafedh Y. Effects of dietary protein on growth and body composition of Nile tilapia (Orechromis niloticus). Aquacult Res. 1999;30:385-393.

Hernández M, Egea M, Rueda F, Aguado F, Martínez F, Garcia B. Effects of commercial diets with different $\mathrm{P} / \mathrm{E}$ ratios on sharpsnout seabream (Diplodus puntazzo) growth and nutrient utilization. Aquacult. 2001;195:321-329.

Kim K, Wang S, Choi G, Park-Bai C. Evaluation of optimum dietary protein-to-energy ratio in juvenile olive flounder Paralichthys olivaceus (Temminck et Schlegel). Aquacult. feeds \& foods nutrition. 2004;35:250-255.

Lee S, Kim K. Effect of various levels of lipid exchanged with dextrin at different protein level in diet on growth and body composition of juvenile flounder Paralichthys olivaceus. Aquacult Nutr. 2005; 11:435-442.

Miller C, Davis D, Phelps R. The effects of dietary protein and lipid on growth and body composition of juvenile and sub-adult red snapper, Lutjanus campechanus (Poey,1860). Aquacult Res. 2005;36:52-60.

Morillo M, Visbal T, Rial L, Ovalles F, Aguirre P, Medina A. Alimentación de alevines de Colossoma macroporum con dietas a base de Erythrina edulis y soya. Rev. Interciencia 2013;38(2):121128.

National Research Council. 1993. Nutrient requirements of Fish. National Academic Press, Washington DC,114 p.
Ozorio R, Valente L, Pousao P, Oliva A. Growth performance and body composition of white sea bream (Diplodus sargus) juveniles fed diets with different protein and lipid levels. Aquacult Res. 2006;37:255-263.

Quintero LG, Pardo BS, Quintero AMC. 2011. Manual técnico para la producción de peces de consumo a pequeña y mediana escala en el departamento de Cundinamarca. Produmedios, Bogotá, DC. 88 p.

Salinas A. 2018. Niveles de energía y relación proteína a energía sobre el desempeño productivo y composición corporal del paco (Piaractus brachypomus). Tesis de maestría en nutrición. Universidad Nacional Agraria La colina. Lima- Perú, 69 p.

Sampaio A, Kubitza F, Cyrino J. Relação energia: proteína na nutrição do tucunaré. Scientia Agricola. 2000;57(2):213-219.

Vásquez W, Pereira M, Arias J. Estudos para composiçăo de uma dieta referência semipurificada para avaliçăo de exigências nutricionais em juvenis de pirapitinga, Piaractus brachypomus (Cuvier, 1818). Rev Brasil Zootecn. 2002;31:283-292.

Vásquez W. 2004. Principios de nutrición aplicada al cultivo de peces. Ed. Universidad de los Llanos. Instituto de Agricultura. 64 p.

Vásquez W, Pereira M, Arias J. Optimum dietary crude protein requirement for juvenile cachama Piaractus brachypomus. Ciencia Rural, Santa María. 2011;41(12):2183-2189.

Vásquez W, Yossa M, Gutiérrez M. Digestibilidad aparente de ingredientes de origen vegetal y animal en la cachama. Pesq Agropec. 2013;48(1):920-927. https://doi.org/10.1590/S0100204X2013000800016.

Vinicius M, Machado D. Exigencia Proteica e Relação Energía/Proteína para Alevinos de Piracanjuba (Brycon orbignyanus). Revista Acuicultura. 2002;1:1-10.

Williams K, Barlow G, Rodgers L, Hockings I, Agcopra C, Ruscoe I. Asian seabass Lates calcarifer perform well when fed pelleted diets high in protein and lipid. Aquacult. 2003;225:191-206. 